\title{
Exact dynamical decay rate for the almost Mathieu operator
}

\author{
Svetlana Jitomirskaya, Helge Krüger, and Wencai LiU
}

We prove that the exponential decay rate in expectation is well defined and is equal to the Lyapunov exponent, for supercritical almost Mathieu operators with Diophantine frequencies.

\section{Introduction}

In physics literature, Lyapunov exponent is often referred to as the inverse localization length, and its positivity is often considered a manifestation of localization in a $1 \mathrm{D}$ system. At the same time, various physically desirable conclusions, such as e.g. the exponential decay of the two-point function at the ground state and positive temperatures with correlation length staying uniformly bounded as temperature goes to zero, are often implicitly assumed as attributes of localization. A way to derive them currently requires a strong form of dynamical localization [3]: the exponential (in space) rate of decay of the two point function, that is

$$
\mathbb{E} \sum_{s}\left|\varphi_{s}(\ell)\right|\left|\varphi_{s}(k)\right| \leq C e^{-\gamma|k-\ell|}
$$

where $\left\{\varphi_{s}\right\}_{s}$ is a complete set of orthonormalized eigenfunctions (and the sum may be localized in energy, if needed).

In view of this, the exponential decay rate in expectation was defined in 14] as

$$
\gamma_{+}:=\limsup _{k \rightarrow \infty}\left(-\frac{\ln \mathbb{E}\left(\sum_{s}\left|\varphi_{s}(0)\right| \cdot\left|\varphi_{s}(k)\right|\right)}{|k|}\right)
$$

and

$$
\gamma_{-}:=\liminf _{k \rightarrow \infty}\left(-\frac{\ln \mathbb{E}\left(\sum_{s}\left|\varphi_{s}(0)\right| \cdot\left|\varphi_{s}(k)\right|\right)}{|k|}\right) .
$$


It is obviously connected to the minimal inverse correlation length. This definition can be localized to an energy range by summing over the eigenfunctions with energies falling in the range, in which case it is linked to the minimal inverse correlation length for Fermi energies falling in that range.

It is well known that there is a long road from positive Lyapunov exponents to a statement like (1). First, positive Lyapunov exponents don't even imply pure point spectrum for a.e. phase [6]. Even for models with positive Lyapunov exponents and known pure point spectrum, dynamical localization may not hold [9], and then an averaged statement (dubbed strong dynamical localization) is strictly stronger, and a result such as (1) is stronger yet (albeit equivalent in all known examples so far).

Yet it may be natural to expect that there is a certain reason to physicists' jump in conclusions, and that for physically relevant models Lyapunov exponent is indeed related to $\gamma_{ \pm}$.

In this paper we prove the first such result.

It turns out that for almost Mathieu operators, arguably the most popular 1D model in physics, the Lyapunov exponent precisely defines the dynamical decay rate.

We define the almost Mathieu operator by its action on $u \in \ell^{2}(\mathbb{Z})$,

$$
\left(H_{\lambda, \alpha, \theta} u\right)(n)=u(n+1)+u(n-1)+V_{\lambda, \alpha, \theta}(n) u(n),
$$

with the potential $V_{\lambda, \alpha, \theta}$ given by

$$
V_{\lambda, \alpha, \theta}(n)=2 \lambda \cos 2 \pi(\theta+n \alpha),
$$

where $\lambda \neq 0$ is the coupling, $\alpha \in \mathbb{R} \backslash \mathbb{Q}$ is the frequency, and $\theta \in \mathbb{R}$ is the phase.

We say that frequency $\alpha$ is Diophantine if there exist $\kappa>0$ and $\tau>0$ such that for $k \neq 0$,

$$
\|k \alpha\|_{\mathbb{R} / \mathbb{Z}} \geq \frac{\tau}{|k|^{\kappa}},
$$

where $\|x\|_{\mathbb{R} / \mathbb{Z}}=\inf _{\ell \in \mathbb{Z}}|x-\ell|$.

Let $L:=\max \{0, \ln |\lambda|\}$ be the Lyapunov exponent of the almost Mathieu operator for energies in the spectrum [8]. We have

Theorem 1.1. Let $|\lambda|>1$, and $\alpha$ be Diophantine. Then

$$
\gamma_{+}=\gamma_{-}=L
$$


Remark 1.2. We define $\gamma_{ \pm}$only in the regime of localization, but of course it is natural to set $\gamma_{ \pm}=0$ in absence of localization. With this definition Theorem 1.1 holds also without the asumption $|\lambda|>1$.

Without loss of generality, we assume $\lambda>0$. We note that almost Mathieu operators have Anderson localization with eigenfunctions decaying exactly at the Lyapunov rate if and only if $\lambda>1$, and $\alpha$ is Diophantine [15, thus we establish equality of the exponential decay rate in expectation and the Lyapunov exponent throughout this entire regime ${ }^{1}$.

Previous quantum dynamics results in the regime of localization have been limited to lower bounds for related quantities, for any model. Bounds for the supercritical (that is $\lambda>1$ ) almost Mathieu operator go back to [12, 21]. Dynamical localization for general analytic quasiperiodic potentials was obtained in [7].

A lower bound on $\gamma_{-}$, establishing its positivity, was proved, under the same assumptions as in Theorem 1.1, in [14]. Previously, lower bounds on $\gamma_{-}$were obtained for the Anderson model, i.e. for the potential being independent identically distributed random variables, in [10, 22] for the one-dimensional case and in [1, 4] for higher dimensions throughout the regimes where corresponding proofs of localization work, thus excluding e.g. Bernoulli. The corresponding result for continuum operators was proven in [2]. Recently, a proof of such lower bound was obtained for an arbitrary 1D bounded Anderson model in [1] using a more delicate implementation of the method of [19] and some ideas of [14].

While lower bounds on $\gamma_{-}$are a corollary of localization, that is of taming the resonances, upper bounds on $\gamma_{+}$are a corollary of delocalization, that is of exploiting the presence of the resonances. It is well known that the latter task is usually harder. In this paper we achieve this, at the same time making both estimates sharp. Our analysis uses (a small part of the) delicate estimates on eigenfunctions obtained in [16]. The statements we need that are similar to those in [16] are presented in the appendix, while the body of the paper consists of the new argument needed to derive the sharp upper and lower bounds. The technique we develop to obtain sharp estimates is also an important ingredient in the upcoming work [17].

\footnotetext{
${ }^{1}$ More precisely, exact Lyapunov decay of the eigenfunctions holds if and only if $\lambda>1$, and limsup $\frac{\ln q_{n+1}}{q_{n}}=0$, where $q_{n}$ are denominators of continued fraction approximants of $\alpha[15]^{q_{n}}$. Our result depends on Lemmas from [16] that were formulated there for the standard Diophantine condition, but our proof would hold for the entire regime $\lim \sup \frac{\ln q_{n+1}}{q_{n}}=0$ if those lemmas were correspondingly upgraded, which is a technical matter.
} 
It is tempting to conjecture that Theorem 1.1 has a universal nature, but one should be cautious. For example, we do not expect it to hold even for weakly Liouville almost Mathieu operators for which localization has been established in [5, 15], with eigenfunctions decaying exponentially but at a non-Lyapunov rate [15. However, even for those a statement of the form $\gamma_{+}=L$ may be plausible. Moreover, almost Mathieu operators are special in that their Lyapunov exponent is constant on the spectrum, and without this condition the statement of the theorem doesn't even make sense. Yet, it is natural to expect that in many physically relevant situations it should be true that $\gamma_{ \pm}=L_{ \pm}$, where $L_{+}=\sup L(E)\left(L_{-}=\inf L(E)\right)$ over $E$ in the spectrum. For example, it is a very interesting question to establish such a connection for the Anderson model where eigenfunctions do decay at the Lyapunov rate (e.g. [19]) as well as in the other models where there is Lyapunov decay of the eigenfunctions. In the framework of the method of [11, 19] this would require more delicate estimates on the probabilities of large deviation sets.

\section{Preliminaries}

In the following, we will consider $\lambda>1$ and $\alpha$ Diophantine fixed, and so set $H_{\theta}:=H_{\lambda, \alpha, \theta}$. We know that for almost every $\theta$, the spectrum of $H_{\theta}$ is pure point [20]. We denote by $\phi_{\theta ; s}$ an orthonormal basis consisting of eigenfunctios of $H_{\theta}$, where the enumeration can be assumed to be measurable [13]. Let $n_{\theta ; s}$ be the position of the leftmost maximum of $\phi_{\theta ; s}$, so

$$
\left|\phi_{\theta ; s}\left(n_{\theta ; s}\right)\right|=\left\|\phi_{\theta ; s}\right\|_{\ell^{\infty}(\mathbb{Z})} .
$$

A key step in the proof of Theorem 1.1 will be to prove the following localization result. Below $\varepsilon$ is always small.

Theorem 2.1. Let $\lambda>1, \alpha$ Diophantine, $\theta \in \mathbb{R}, \ell \in \mathbb{Z}$, and $\ell^{\prime}=\left|\ell-n_{\theta ; s}\right|$. Let $x_{0} \in\left[-2 \ell^{\prime}, 2 \ell^{\prime}\right]$ be such that

$$
\left|\sin \pi\left(2 \theta+\alpha\left(2 n_{\theta ; s}+x_{0}\right)\right)\right|=\min _{|x| \leq 2 \ell^{\prime}}\left|\sin \pi\left(2 \theta+\alpha\left(2 n_{\theta ; s}+x\right)\right)\right| .
$$

Then for large $\ell^{\prime}$ (depending on $\varepsilon$ ) we have

- if $\ell$ and $x_{0}+n_{\theta ; s}$ are on different sides of $n_{\theta ; s}$, that is $\left(\ell-n_{\theta ; s}\right) x_{0}<0$, then

$$
\left|\phi_{\theta ; s}(\ell)\right| \leq e^{-(L-\varepsilon)\left|\ell-n_{\theta ; s}\right|}\left|\phi_{\theta ; s}\left(n_{\theta ; s}\right)\right| \text {. }
$$


- if $\left(\ell-n_{\theta ; s}\right) x_{0} \geq 0$ and $\left|\sin \pi\left(2 \theta+\alpha\left(2 n_{\theta ; s}+x_{0}\right)\right)\right| \geq e^{-\eta\left|\ell-n_{\theta ; s}\right|}$ for some $\eta \in(0, L-\varepsilon)$, then

$$
\left|\phi_{\theta ; s}(\ell)\right| \leq e^{-(L-\varepsilon-\eta)\left|\ell-n_{\theta ; s}\right|}\left|\phi_{\theta ; s}\left(n_{\theta ; s}\right)\right| .
$$

Proof. Theorem 2.1 is obtained using the arguments from [16]. We include a proof in the appendix.

Theorem 2.1 implies the following corollary immediately.

Corollary 2.2. Let $\lambda>1, \alpha$ Diophantine, $\theta \in \mathbb{R}, \ell \in \mathbb{Z}$, and $\ell^{\prime}=\left|\ell-n_{\theta ; s}\right|$. Let $x_{0} \in\left[-2 \ell^{\prime}, 2 \ell^{\prime}\right]$ such that

$$
\left|\sin \pi\left(2 \theta+\alpha\left(2 n_{\theta ; s}+x_{0}\right)\right)\right|=\min _{|x| \leq 2 \ell^{\prime}}\left|\sin \pi\left(2 \theta+\alpha\left(2 n_{\theta ; s}+x\right)\right)\right| .
$$

Suppose for some $\eta \in(0, L-\varepsilon)$

$$
\min _{|x| \leq 2 \ell^{\prime}}\left|\sin \pi\left(2 \theta+\alpha\left(2 n_{\theta ; s}+x\right)\right)\right|>e^{-\eta\left|\ell-n_{\theta ; s}\right|} .
$$

Then we have

$$
\left|\phi_{\theta ; s}(\ell)\right| \leq e^{-(L-\eta-\varepsilon)\left|\ell-n_{\theta ; s}\right|}\left|\phi_{\theta ; s}\left(n_{\theta ; s}\right)\right|
$$

\section{The lower bound}

In this part we will prove the lower bound in Theorem $1.1, \gamma_{-} \geq L$. That is we will fix $\ell \in \mathbb{Z}$ and bound

$$
\int_{0}^{1} \sum_{s}\left|\phi_{\theta ; s}(0) \phi_{\theta ; s}(\ell)\right| d \theta=\sum_{n \in \mathbb{Z}} \int_{0}^{1} \sum_{n_{\theta ; s}=n}\left|\phi_{\theta ; s}(0) \phi_{\theta ; s}(\ell)\right| d \theta
$$

from above. By orthogonality, we have for any $s$,

$$
\sum_{n}\left|\phi_{\theta ; s}(n)\right|^{2}=1
$$

and for any $\theta \in \mathbb{R}$

$$
\sum_{s}\left|\phi_{\theta ; s}(n)\right|^{2}=1
$$

By symmetry, we can clearly assume that $\ell \geq 0$. We note that in order to prove the lower bound in Theorem 1.1, it suffices to show 
Theorem 3.1. Let $\lambda>1, \alpha$ Diophantine, and $0<\Gamma<L$. Then for $\ell \geq 0$ large enough, we have

$$
\sum_{n \in \mathbb{Z}} \int_{0}^{1} \sum_{n_{\theta ; s}=n}\left|\phi_{\theta ; s}(0) \phi_{\theta ; s}(\ell)\right| d \theta \leq e^{-\Gamma \ell} .
$$

For $n \in \mathbb{Z}$ and $0<\eta<L$, we define the sets

$$
A_{\eta ; n}=\left\{\theta: \min _{\left|n^{\prime}\right| \leq 10|n|}\left|\sin \pi\left(2 \theta+\alpha\left(2 n+n^{\prime}\right)\right)\right| \leq e^{-\eta|n|}\right\}
$$

and

$$
B_{\eta ; n ; \ell}=\left\{\theta: \min _{\left|n^{\prime}\right| \leq 10|n-\ell|}\left|\sin \pi\left(2 \theta+\alpha\left(2 n+n^{\prime}\right)\right)\right| \leq e^{-\eta|n-\ell|}\right\}
$$

We clearly have that $\left|A_{\eta, n}\right| \leq(20|n|+1) e^{-\eta|n|}$ and $\left|B_{\eta ; n ; \ell}\right| \leq(20|n-\ell|+$ 1) $e^{-\eta|n-\ell|}$.

By Theorem 2.1 and Corollary 2.2, we can obtain the following Lemma.

Lemma 3.2. For any $\eta \in(0, L-\varepsilon)$, the following estimates hold,

(i) For $\theta \notin A_{\eta ; n}$ and $n_{\theta ; s}=n$, we have

$$
\left|\phi_{\theta ; s}(0)\right| \leq e^{-(L-\eta-\varepsilon)|n|}\left|\phi_{\theta ; s}(n)\right|
$$

for large $|n|$.

(ii) For $\theta \notin B_{\eta ; n ; \ell}$ and $n_{\theta ; s}=n$, we have

$$
\left|\phi_{\theta ; s}(\ell)\right| \leq e^{-(L-\eta-\varepsilon)|n-\ell|}\left|\phi_{\theta ; s}(n)\right|,
$$

for large $|n-\ell|$.

Proof of Theorem 3.1. Let $\delta_{0}$ be a small positive constant. We write

$$
\begin{aligned}
\sum_{n \in \mathbb{Z}} \int_{0}^{1} \sum_{n_{\theta ; s}=n}\left|\phi_{\theta ; s}(0) \phi_{\theta ; s}(\ell)\right| d \theta & =\sum_{\left(1-\delta_{0}\right) \ell}^{+\infty}+\sum_{-\infty}^{\delta_{0} \ell}+\sum_{n=\delta_{0} \ell}^{\left(1-\delta_{0}\right) \ell} \\
& =\mathrm{I}+\mathrm{II}+\mathrm{III} .
\end{aligned}
$$

We estimate I first. In this case, fix $n_{\theta ; s}=n \geq\left(1-\delta_{0}\right) \ell$. By (i) of Lemma 3.2 and $(16)$, we can conclude that for any $n \geq\left(1-\delta_{0}\right) \ell$ and 
$\theta \notin A_{\eta ; n}$,

$$
\begin{aligned}
\sum_{n_{\theta ; s}=n}\left|\phi_{\theta ; s}(0) \phi_{\theta ; s}(\ell)\right| & \leq \sum_{n_{\theta ; s}=n}\left|\phi_{\theta ; s}(0) \phi_{\theta ; s}(n)\right| \\
& \leq e^{-(L-\eta-\varepsilon) n} \sum_{n_{\theta ; s}=n}\left|\phi_{\theta ; s}(n)\right|^{2} \\
& \leq e^{-(L-\eta-\varepsilon) n}
\end{aligned}
$$

Therefore, we have that for $t=e^{\eta n} e^{-(L-\varepsilon) n}$ and $\eta \in(0, L-2 \varepsilon)$,

$$
\left\{\theta \in \mathbb{T}: \sum_{n_{\theta ; s}=n}\left|\phi_{\theta ; s}(0) \phi_{\theta ; s}(\ell)\right|>t\right\} \subseteq A_{\eta ; n}
$$

Let $t_{1}=e^{-\varepsilon n}, t_{2}=e^{-(L-2 \varepsilon) n}$. Define $\eta(t)$ for $t_{2} \leq t \leq 1$ implicitly by $t=$ $e^{\eta(t) n} \cdot e^{-(L-\varepsilon) n}$. Then for $t_{2} \leq t \leq 1, \eta(t) \geq \varepsilon$, and we have

$$
\left|A_{\eta(t) ; n}\right| \leq(20 n+1) e^{-(L-\varepsilon) n} / t .
$$

Since $\sum_{n_{\theta ; s}=n}\left|\phi_{\theta ; s}(0) \phi_{\theta ; s}(\ell)\right| \leq 1$, for any Borel $\Omega \in \mathbb{T}$, we have

$$
\int_{\Omega} \sum_{n_{\theta ; s}=n}\left|\phi_{\theta ; s}(0) \phi_{\theta ; s}(\ell)\right| d \theta=\int_{[0,1]}\left|\left\{\theta \in \Omega: \sum_{n_{\theta ; s}=n}\left|\phi_{\theta ; s}(0) \phi_{\theta ; s}(\ell)\right|>t\right\}\right| d t .
$$

Thus we have

$$
\int_{0}^{1} \sum_{n_{\theta ; s}=n}\left|\phi_{\theta ; s}(0) \phi_{\theta ; s}(\ell)\right| d \theta=\int_{0}^{t_{2}}+\int_{t_{2}}^{t_{1}}+\int_{t_{1}}^{1}=i+i i+i i i .
$$

Then

$$
i \leq t_{2} \leq e^{-(L-2 \varepsilon) n}
$$

From (24), 22) and (23), one has for large $n$,

$$
\begin{aligned}
i i & \leq \int_{t_{2}}^{t_{1}}\left|A_{\eta(t) ; n}\right| d t \\
& \leq \int_{t_{2}}^{t_{1}}(20|n|+1) e^{-(\ln \lambda-\varepsilon) n} / t d t \\
& \leq e^{-(L-2 \varepsilon) n} .
\end{aligned}
$$


Noticing that $\left|A_{\eta\left(t_{1}\right) ; n}\right| \leq(20|n|+1) e^{-(\ln \lambda-2 \varepsilon) n}$, one has

$$
i i i \leq\left(1-t_{1}\right)\left|A_{\eta\left(t_{1}\right) ; n}\right| \leq e^{-(L-3 \varepsilon) n}
$$

Thus, for $n \geq\left(1-\delta_{0}\right) \ell$,

$$
\int_{0}^{1} \sum_{n_{\theta ; s}=n}\left|\phi_{\theta ; s}(0) \phi_{\theta ; s}(\ell)\right| d \theta \leq e^{-(L-3 \varepsilon) n}
$$

Then, we have that

$$
\mathrm{I}=\sum_{n=\left(1-\delta_{0}\right) \ell}^{\infty} \int_{0}^{1} \sum_{n_{\theta ; s}=n}\left|\phi_{\theta ; s}(0) \psi_{\theta ; s}(\ell)\right| d \theta \leq e^{-(L-4 \varepsilon)\left(1-\delta_{0}\right) \ell}
$$

Similarly,

$$
\mathrm{II} \leq e^{-(L-4 \varepsilon)\left(1-\delta_{0}\right) \ell}
$$

Now we are in a position to estimate III. For $\theta \in[0,1] \backslash A_{\delta_{0} ; n} \cup B_{\delta_{0} ; n ; \ell}$, by Lemma 3.2 and 16 , one has

$$
\begin{aligned}
\sum_{n_{\theta ; s}=n}\left|\phi_{\theta ; s}(0) \phi_{\theta ; s}(\ell)\right| & \leq e^{-\left(L-\delta_{0}-\varepsilon\right) \ell} \sum_{n_{\theta ; s}=n}\left|\phi_{\theta ; s}(n)\right|^{2} \\
& \leq e^{-\left(L-\delta_{0}-\varepsilon\right) \ell}
\end{aligned}
$$

It leads to

(32)

$$
\sum_{\delta_{0} \ell \leq n \leq\left(1-\delta_{0}\right) \ell} \int_{[0,1] \backslash\left(A_{\delta_{0} ; n} \cup B_{\delta_{0} ; n ; \ell}\right)} \sum_{n_{\theta ; s}=n}\left|\phi_{\theta ; s}(0) \phi_{\theta ; s}(\ell)\right| d \theta \leq e^{-\left(L-\delta_{0}-2 \varepsilon\right) \ell} .
$$

For $\theta \in A_{\delta_{0} ; n} \cup B_{\delta_{0} ; n ; \ell}$, let $x_{0}(\theta) \in[-10 \ell, 10 \ell]$ be such that

$$
\left|\sin \pi\left(2 \theta+\alpha x_{0}\right)\right|=\min _{|x| \leq 10 \ell}|\sin \pi(2 \theta+\alpha x)| .
$$

Notice that $x_{0}$ is unique by the fact that $\alpha$ satisfies Diophantine condition. 
Let

$$
\Omega_{1}=\left\{\theta \in A_{\delta_{0} ; n} \cup B_{\delta_{0} ; n ; \ell} \mid x_{0}(\theta)<n\right\},
$$

and

$$
\Omega_{2}=\left\{\theta \in A_{\delta_{0} ; n} \cup B_{\delta_{0} ; n ; \ell} \mid x_{0}(\theta) \geq n\right\} .
$$

By Theorem 2.1 and the fact that $\delta_{0} \ell \leq n \leq\left(1-\delta_{0}\right) \ell$, for any $\theta \in \Omega_{1}$,

$$
\left|\phi_{\theta ; s}(\ell)\right| \leq e^{-(L-\varepsilon)|\ell-n|}\left|\phi_{\theta ; s}(n)\right|
$$

and for any $\theta \in \Omega_{2}$,

$$
\left|\phi_{\theta ; s}(0)\right| \leq e^{-(L-\varepsilon)|n|}\left|\phi_{\theta ; s}(n)\right|
$$

For $\theta \in \Omega_{1} \backslash A_{\eta ; n}$ with $\delta_{0}<\eta<\ln L-\varepsilon$, by Lemma 3.2 , we have that

$$
\begin{aligned}
\sum_{n_{\theta ; s}=n}\left|\phi_{\theta ; s}(0) \phi_{\theta ; s}(\ell)\right| & \leq e^{-(L-\varepsilon)|n-\ell|} e^{-(L-\eta-\varepsilon)|n|} \sum_{n_{\theta ; s}=n}\left|\phi_{\theta ; s}(n)\right|^{2} \\
& \leq e^{-(L-\varepsilon)|n-\ell|} e^{-(L-\eta-\varepsilon)|n|} \\
& \leq e^{-(L-\varepsilon) \ell} e^{\eta|n|}
\end{aligned}
$$

A similar bound holds for $\theta \in \Omega_{2} \backslash B_{\eta ; n ; \ell}$. That is, for $\theta \in \Omega_{2} \backslash B_{\eta ; n ; \ell}$ and $\delta_{0}<\eta<L-\varepsilon$,

$$
\sum_{n_{\theta ; s}=n}\left|\phi_{\theta ; s}(0) \phi_{\theta ; s}(\ell)\right| \leq e^{-(L-\varepsilon) \ell} e^{\eta|n|}
$$

By $34,35,24$ and 23 , we then have 25 with $\int_{0}^{1}$ replaced by $\int_{\Omega_{1} \cup \Omega_{2}}$ and also (26), 27), 28). Thus we also have

$$
\int_{\Omega_{1} \cup \Omega_{2}} \sum_{n_{\theta ; s}=n}\left|\phi_{\theta ; s}(0) \phi_{\theta ; s}(\ell)\right| d \theta \leq e^{-(L-\varepsilon) \ell} .
$$

It leads to

$$
\sum_{\delta_{0} \ell \leq n \leq\left(1-\delta_{0}\right) \ell} \int_{\Omega_{1} \cup \Omega_{2}} \sum_{n_{\theta ; s}=n}\left|\phi_{\theta ; s}(0) \phi_{\theta ; s}(\ell)\right| d \theta \leq e^{-(L-2 \varepsilon) \ell} .
$$

By 32 and $(36)$, we get the bound of II,

$$
\mathrm{II} \leq e^{-\left(L-\delta_{0}-3 \varepsilon\right) \ell} .
$$


Putting the bounds of I, II and III together, we have

$$
\sum_{n \in \mathbb{Z}} \int_{0}^{1} \sum_{n_{\theta ; s}=n}\left|\phi_{\theta ; s}(0) \phi_{\theta ; s}(\ell)\right| d \theta \leq e^{-\left(L-\delta_{0}-6 \varepsilon\right) \ell} .
$$

Letting $\delta_{0}, \varepsilon \rightarrow 0$, we obtain Theorem 3.1.

\section{The upper bound}

In this part we will prove the upper bound: $\gamma_{+} \leq L$.

Theorem 4.1. For any $\Gamma$ satisfying $L<\Gamma \leq 2 L$, we have for $n$ large enough

$$
\ln \int_{0}^{1} \sum_{s}\left|\phi_{\theta ; s}(0) \phi_{\theta ; s}(n)\right| d \theta \geq-\Gamma|n| .
$$

Fix $L<\Gamma \leq 2 L$ and large $n$. Define sets

$$
\Theta_{1}=\left\{\theta \in[0,1]: e^{-2 \Gamma|n|} \leq|\sin \pi(2 \theta+n \alpha)| \leq e^{-\Gamma|n|}\right\}
$$

and

$$
\begin{aligned}
\Theta_{2}=\{\theta \in[0,1] & \text { there exists some }|k| \geq 1000|n| \\
& \text { such that } \left.|\sin \pi(2 \theta+k \alpha)| \leq e^{-\frac{L}{100}|k|}\right\}
\end{aligned}
$$

Then $\Theta=\Theta_{1} \backslash \Theta_{2}$ has measure satisfying $|\Theta| \geq \frac{1}{100} e^{-\Gamma|n|}$.

Lemma 4.2. Let $\alpha$ be Diophantine with constants $\kappa, \tau>0$. Then for any $\theta \in \Theta$ and for any $m>C(\kappa, \tau)|n|$,

$$
\min _{|x| \leq m}|\sin \pi(2 \theta+x \alpha)| \geq e^{-\frac{L}{100}|m|} .
$$

Proof. Let $x_{0}$ be such that the minimum in 40 is attained at $x=x_{0}$. We split our analysis into three cases depending on the value of $x_{0}$.

Case I. $\left|x_{0}\right| \geq 1000|n|$. Then the Lemma holds because of $\theta \notin \Theta_{2}$.

Case II. $\left|x_{0}\right| \leq 1000|n|$ and $x_{0} \neq n$. The Lemma holds because of $\theta \in \Theta_{1}$ and DC frequencies.

Case III. $x_{0}=n$. The Lemma holds because of $\theta \in \Theta_{1}$ (using $\mid \sin \pi(2 \theta+$ $\left.n \alpha) \mid \geq e^{-2 \Gamma|n|}\right)$. 
It clearly suffices to show that for the eigenfunctions $\phi_{s}$ of $H=H_{\lambda, \alpha, \theta}$ (we ignore the dependence on $\theta$ ) we have

$$
\sum_{s}\left|\phi_{s}(0) \phi_{s}(n)\right| \geq \frac{1}{2}
$$

as long as $|n|$ is large enough, uniformly in $\theta \in \Theta$. The first step is

Proposition 4.3. For $|n|$ large enough and $\theta \in \Theta$, we have

$$
\sum_{|m| \leq C_{\star}|n|} \sum_{n_{s}=m}\left|\phi_{s}(0)\right|^{2} \geq \frac{1}{2},
$$

where $C_{\star}=C(\kappa, \tau)$.

Proof. Without loss of generality, assume $n \geq 0$. Suppose $m \leq-C_{\star} n$ or $m \geq$ $C_{\star} n$.

Using Corollary 2.2 with $n_{\theta ; s}=m, \ell=0$, by 40 , we have

$$
\left|\phi_{s}(0)\right| \leq\left|\phi_{s}(m)\right| e^{-\frac{L}{2}|m|}
$$

Thus

$$
\begin{aligned}
\sum_{|m| \geq C_{\star} n} \sum_{n_{s}=m}\left|\phi_{s}(0)\right|^{2} & \leq \sum_{|m| \geq C_{\star} n} \sum_{n_{s}=m}\left|\phi_{s}(m)\right|^{2} e^{-L|m|} \\
& =\sum_{|m| \geq C_{\star} n} e^{-L|m|} \sum_{n_{s}=m}\left|\phi_{s}(m)\right|^{2} \\
& \leq \sum_{|m| \geq C_{\star} n} e^{-L|m|} \\
& \leq \frac{1}{2}
\end{aligned}
$$

Combining with (16), the result follows.

The following lemma is similar to a statement appearing in [16] with some modifications. We present a proof in the Appendix. 
Lemma 4.4. Suppose

$$
|\sin \pi(2 \theta+n \alpha)| \leq e^{-\Gamma|n|}
$$

with $L<\Gamma \leq 2 L$. Suppose $\phi$ is an $\ell^{2}$ solution of $H_{\lambda, \alpha, \theta} \phi=E \phi$. Then

$$
|\phi(n)-\phi(0)| \leq e^{-\frac{1}{2}(\Gamma-L-\varepsilon)|n|}\|\phi\|_{\ell^{\infty}(\mathbb{Z})}
$$

Proof of Theorem 4.1. For large n, by Proposition 4.3 and Lemma 4.4, one has for $\theta \in \Theta$,

$$
\begin{aligned}
\sum_{s}\left|\phi_{s}(0) \phi_{s}(n)\right| \geq & \sum_{|m| \leq C_{\star}|n|} \sum_{n_{s}=m}\left|\phi_{s}(0) \phi_{s}(n)\right| \\
\geq & \sum_{|m| \leq C_{\star}|n|} \sum_{n_{s}=m}\left|\phi_{s}(0)\right|\left(\left|\phi_{s}(0)\right|-e^{-\frac{1}{2}(\Gamma-L-\varepsilon)|n|}|| \phi_{s} \mid \ell_{\ell^{\infty}(\mathbb{Z})}\right. \\
\geq & \sum_{|m| \leq C_{\star}|n|} \sum_{n_{s}=m}\left|\phi_{s}(0)\right|^{2} \\
& -e^{-\frac{1}{2}(\Gamma-L-\varepsilon)|n|} \sum_{|m| \leq C_{\star}|n| n_{s}=m}\left|\phi_{s}(0)\right|\left(\sum_{|k| \leq C_{\star}|n|}\left|\phi_{s}(k)\right|^{2}\right)^{\frac{1}{2}} \\
\geq & \frac{1}{2}-\left.2 e^{-\frac{1}{2}(\Gamma-L-\varepsilon)|n|} \sum_{|m| \leq C_{\star}|n| n_{s}=m}|k| k \phi_{s}(k)\right|^{2} \\
\geq & \frac{1}{4} .
\end{aligned}
$$

Then

$$
\begin{aligned}
\int_{0}^{1} \sum_{s}\left|\phi_{s}(0) \phi_{s}(n)\right| d \theta & \geq \int_{\Theta} \sum_{s}\left|\phi_{s}(0) \phi_{s}(n)\right| d \theta \\
& \geq \frac{e^{-\Gamma|n|}}{400}
\end{aligned}
$$

This implies Theorem 4.1 .

\section{Appendix A. Proof of Theorem 2.1}

By shifting the operator by $n_{\theta ; s}$ units we can assume $n_{\theta ; s}=0$. Without loss of generality, we assume $\ell>n_{\theta ; s}$. Then in order to prove Theorem 2.1, it suffices to prove the following theorem. 
Theorem A.1. Let $\lambda>1, \alpha$ Diophantine, $n_{\theta ; s}=0, \phi_{s}(0)=1, \ell \in \mathbb{Z}^{+}$. Let $x_{0} \in[-2 \ell, 2 \ell]$ be such that

$$
\left|\sin \pi\left(2 \theta+\alpha x_{0}\right)\right|=\min _{|x| \leq 2 \ell}|\sin \pi(2 \theta+\alpha x)| .
$$

Then the following statements hold for large $\ell$ :

If $x_{0} \in[-2 \ell, 0]$, then

$$
\left|\phi_{s}(\ell)\right| \leq e^{-(L-\varepsilon) \ell}
$$

If for $\eta \in(0, L-\varepsilon)$

$$
\min _{|x| \leq 2 \ell}|\sin \pi(2 \theta+\alpha x)|>e^{-\eta \ell}
$$

and $x_{0} \in[0,2 \ell]$, then

$$
\left|\phi_{s}(\ell)\right| \leq e^{-(L-\eta-\varepsilon) \ell}
$$

Suppose $H_{\lambda, \alpha, \theta} \varphi=E \varphi$. Let $U^{\varphi}(y)=\left(\begin{array}{c}\varphi(y) \\ \varphi(y-1)\end{array}\right)$. It isa standard fact (e.g. (37) in [16]) that for large $\left|k_{1}-k_{2}\right|$,

$$
C e^{-(L+\varepsilon)\left|k_{1}-k_{2}\right|}|| U^{\varphi}\left(k_{2}\right)\|\leq\| U^{\varphi}\left(k_{1}\right)\left\|\leq C e^{(L+\varepsilon)\left|k_{1}-k_{2}\right|}|| U^{\varphi}\left(k_{2}\right)\right\| .
$$

Lemma A.2. [16, Lemma 3.4] Let $r_{y}^{\varphi}=\max _{|\sigma| \leq 10 \gamma}|\varphi(y+\sigma k)|$. Suppose $k_{0} \in[-2 C k, 2 C k]$ is such that

$$
\left|\sin \pi\left(2 \theta+\alpha k_{0}\right)\right|=\min _{|x| \leq 2 C k}|\sin \pi(2 \theta+\alpha x)|,
$$

where $C \geq 1$ is a constant. Let $\gamma, \varepsilon$ be small positive constants. Let $y_{1}=$ $0, y_{2}=k_{0}, y_{3} \in[-2 C k, 2 C k]$.Assume y lies in $\left[y_{i}, y_{j}\right]$ (i.e., $\left.y \in\left[y_{i}, y_{j}\right]\right)$ with $\left|y_{i}-y_{j}\right| \geq k$ and $y_{s} \notin\left[y_{i}, y_{j}\right], s \neq i, j$. Suppose $\left|y_{i}\right|,\left|y_{j}\right| \leq C k$ and $\left|y-y_{i}\right| \geq$ $10 \gamma k,\left|y-y_{j}\right| \geq 10 \gamma k$. Then for large enough $k$,

$$
\begin{array}{r}
r_{y}^{\varphi} \leq \max \left\{r_{y_{i}}^{\varphi} \exp \left\{-(L-\varepsilon)\left(\left|y-y_{i}\right|-3 \gamma k\right)\right\}\right. \\
\left.r_{y_{j}}^{\varphi} \exp \left\{-(L-\varepsilon)\left(\left|y-y_{j}\right|-3 \gamma k\right)\right\}\right\} .
\end{array}
$$


Lemma A.3. [16, Lemma 3.7] Fix $0<t<$ L. Suppose

$$
|\sin \pi(2 \theta+\alpha k)|=e^{-t|k|} .
$$

Then for large $|k|$

$$
\left\|U^{\varphi}(k)\right\| \leq \max \left\{\left\|U^{\varphi}(0)\right\|,\left\|U^{\varphi}(2 k)\right\|\right\} e^{-(L-t-\varepsilon)|k|} .
$$

Proof of Theorem A.1. We start with the proof of Case I. Let $\varphi=\phi$, $\gamma=\varepsilon, \quad k=\ell, \quad C=1, \quad k_{0}=x_{0}<0$ and $y_{3}=2 \ell$ in Lemma A.2. By Lemma A.2, one has $\ell \in\left[y_{1}, y_{3}\right]$ and $y_{2}<y_{1}$, so

$$
r_{\ell}^{\phi} \leq e^{-(L-C \varepsilon) \ell} r_{0}^{\phi}+e^{-(L-C \varepsilon) \ell} r_{2 \ell}^{\phi} \leq e^{-(L-C \varepsilon) \ell},
$$

since $|\phi(n)| \leq 1$ for all $n \in \mathbb{Z}$. By A.5 and A.9, we have

$$
|\phi(\ell)| \leq e^{-(L-C \varepsilon) \ell} .
$$

It finishes the proof of Case I.

Now we turn to Case II. Let $t$ be such that $t x_{0}=\eta \ell$. Let $\varphi=\phi, \gamma=$ $\varepsilon, k=\ell, C=1, k_{0}=x_{0}>0$ and $y_{3}=2 \ell$ in Lemma A.2. By Lemma A.2 and A.5), one has (as in the proof of Case I), one has

$$
|\phi(\ell)| \leq e^{-(L-\varepsilon) \ell}+e^{-(L-\varepsilon)\left|\ell-x_{0}\right|}|| U^{\phi}\left(x_{0}\right) \| .
$$

Suppose $x_{0} \geq\left(\frac{\eta}{L}+\varepsilon\right) \ell$. In this case, by the definition of $t$, one has $0<$ $t<L$. Let $k=x_{0}$ and $\varphi=\phi$ in Lemma A.3, one has

$$
\text { (A.11) }\left\|U^{\phi}\left(x_{0}\right)\right\| \leq \max \left\{\left\|U^{\phi}(0)\right\|,\left\|U^{\phi}\left(2 x_{0}\right)\right\|\right\} e^{-(L-t-\varepsilon) x_{0}} \leq e^{-(L-t-\varepsilon) x_{0}} .
$$

In this case, A.4 follows from A.10) and A.11).

Suppose $0 \leq x_{0} \leq\left(\frac{\eta}{L}+\varepsilon\right) \ell$. In this case, A.4 follows from A.10 directly since $\left\|U^{\phi}\left(x_{0}\right)\right\| \leq 2$.

\section{Appendix B. Proof of Lemma 4.4}

Proof. Without loss of generality, we assume $n>0$. Set $A=\|\phi\|_{\ell^{\infty}(\mathbb{Z})}$. We let $\hat{\phi}(k)=\phi(n-k), V(k)=2 \lambda \cos 2 \pi(\theta+k \alpha)$ and $\hat{V}(k)=2 \lambda \cos 2 \pi(\theta+$ 
$(n-k) \alpha)$. Then by the assumption 43 , one has for all $k \in \mathbb{Z}$,

$$
|V(k)-\hat{V}(k)| \leq C e^{-\Gamma n} .
$$

We also have

$$
\phi(k+1)+\phi(k-1)+V(k) \phi(k)=E \phi(k)
$$

and

$$
\hat{\phi}(k+1)+\hat{\phi}(k-1)+\hat{V}(k) \tilde{\phi}(k)=E \hat{\phi}(k) .
$$

Let $W(n)=W(f, g)=f(n+1) g(n)-f(n) g(n+1)$ be the Wronskian. Let

$$
\hat{U}(k)=\left(\begin{array}{c}
\hat{\phi}(k) \\
\hat{\phi}(k-1)
\end{array}\right),
$$

and

$$
U(k)=\left(\begin{array}{c}
\phi(k) \\
\phi(k-1)
\end{array}\right) .
$$

By a standard calculation using (B.12), (B.13), (B.14) and palindromic arguments as in [18] ${ }^{2}$, we have,

$$
\begin{aligned}
|W(\phi, \hat{\phi})(k)-W(\phi, \hat{\phi})(k-1)| & \leq|V(k)-\hat{V}(k)||\phi(k) \hat{\phi}(k)| \\
& \leq C e^{-L n}|\phi(k) \hat{\phi}(k)| \\
& \leq C A^{2} e^{-\Gamma n}
\end{aligned}
$$

In Lemma A.2, let $k_{0}=n$ and $y_{3}=1000 n$, then by A.6 one has

$$
|U(m-1)|,|U(m)| \leq e^{-\Gamma n} A,
$$

where $m=500 n$.

By B.15 and B.16, we have

$$
|W(\phi, \hat{\phi})(k)| \leq A^{2} e^{-(\Gamma-\varepsilon) n},
$$

for $|k| \leq 500 n$.

Now we split $n$ into cases, depending on whether it is odd or even.

\footnotetext{
${ }^{2}$ Palindromic argument of [18] then yields $\left\|U\left(\frac{n}{2}\right)\right\| \leq e^{-(\Gamma-\varepsilon) \frac{n}{2}}$ if $n$ is even and analogous statement if $n$ is odd. Here we want to gain a factor of $A^{2}$.
} 
Case 1. $n$ is even. Let $m=\frac{n}{2}$, then

$$
U(m)=\left(\begin{array}{c}
\phi(m) \\
\phi(m-1)
\end{array}\right) ; \quad \hat{U}(m)=\left(\begin{array}{c}
\phi(m) \\
\phi(m+1)
\end{array}\right) .
$$

Applying B.17 with $k=m-1$, we have

$$
|\phi(m)||\phi(m+1)-\phi(m-1)| \leq A^{2} e^{-(\Gamma-\varepsilon) n} .
$$

This implies

$$
|\phi(m)| \leq A e^{-\frac{1}{2}(\Gamma-\varepsilon) n},
$$

or

$$
|\phi(m+1)-\phi(m-1)| \leq A e^{-\frac{1}{2}(\Gamma-\varepsilon) n} .
$$

If (B.18) holds, by (B.13), we also have

$$
|\phi(m+1)+\phi(m-1)| \leq A e^{-\frac{1}{2}(\Gamma-\varepsilon) n} .
$$

Putting (B.18) and (B.20) together, we get

$$
\|U(m)+\hat{U}(m)\| \leq A e^{-\frac{1}{2}(\Gamma-\varepsilon) n} .
$$

If B.19 holds, we have

$$
\|U(m)-\hat{U}(m)\| \leq A e^{-\frac{1}{2}(\Gamma-\varepsilon) n}
$$

Thus in case 1 there exists $\iota \in\{-1,1\}$ such that

$$
\|U(m)+\iota \hat{U}(m)\| \leq A e^{-\frac{1}{2}(\Gamma-\varepsilon) n} .
$$

In Lemma A.2, let $k_{0}=n, y_{1}=0$ and $y_{3}=m$, then by A.5 one has,

$$
\|\hat{U}(m)\| \leq A e^{-(L-\varepsilon) m}
$$

Let $T$ and $\hat{T}$ be the transfer matrices associated with potentials $V$ and $\hat{V}$, taking $U(m), \hat{U}(m)$ to $U(0), \hat{U}(0)$ correspondingly. By B.12, the usual 
uniform upper semi-continuity and telescoping, one has

$$
\|T\|,\|\hat{T}\| \leq e^{(L+\varepsilon) m}
$$

and

$$
\|T-\hat{T}\| \leq e^{(L-2 \Gamma+\varepsilon) m}
$$

Then by B.23), we have

$$
\begin{aligned}
\|U(0)+\iota \hat{U}(0)\| & \leq\|T\|\|U(m)+\iota \hat{U}(m)\|+\|T-\hat{T}\|\|\hat{U}(m)\| \\
& \leq A e^{(L+\varepsilon) m} e^{-\frac{1}{2}(L-\varepsilon) n}+A e^{(L-2 \Gamma+\varepsilon) m} e^{-m(L-\varepsilon)} . \\
& \leq A e^{-\frac{1}{2}(\Gamma-L-\varepsilon) n} .
\end{aligned}
$$

This completes the proof for even $n$ due to the definition of $U(0)$ and $\hat{U}(0)$.

Case 2. $n$ is odd. Let $\tilde{m}=\frac{N-1}{2}$, then

$$
U(\tilde{m}+1)=\left(\begin{array}{c}
\phi(\tilde{m}+1) \\
\phi(\tilde{m})
\end{array}\right) ; \quad \hat{U}(\tilde{m}+1)=\left(\begin{array}{c}
\phi(\tilde{m}) \\
\phi(\tilde{m}+1)
\end{array}\right)
$$

Combining with (B.17), we have

$$
|\phi(\tilde{m})+\phi(\tilde{m}+1)||\phi(\tilde{m})-\phi(\tilde{m}+1)| \leq A^{2} e^{-(\Gamma-\varepsilon) n} .
$$

This implies

$$
|\phi(\tilde{m})+\phi(\tilde{m}+1)| \leq A e^{-\frac{1}{2}(\Gamma-\varepsilon) n}
$$

or

$$
|\phi(\tilde{m}+1)-\phi(\tilde{m})| \leq A e^{-\frac{1}{2}(\Gamma-\varepsilon) n} .
$$

Thus in case 2 , there also exists $\iota \in\{-1,1\}$ such that

$$
\|U(\tilde{m}+1)+\iota \hat{U}(\tilde{m}+1)\| \leq A e^{-\frac{1}{2}(\Gamma-\varepsilon) n} .
$$

The rest of the proof is the same as in case 1 .

\section{Acknowledgments}

S.J. was supported by NSF DMS-1401204 and DMS-1901462. W. L. was supported by NSF DMS-1700314/2015683, DMS-2000345, the AMS-Simons Travel Grant 2016-2018 and the Southeastern Conference (SEC) Faculty Travel Grant 2020-2021. S.J. and W.L. are grateful to the Isaac Newton Institute for Mathematical Sciences, Cambridge, for its hospitality, supported 
by EPSRC Grant Number EP/K032208/1, during the 2015 programme Periodic and Ergodic Spectral Problems where an important progress on this work was made.

\section{References}

[1] M. Aizenman, Localization at weak disorder: Some elementary bounds, Rev. Math. Phys. 6 (1994), no. 5, 1163-1182.

[2] M. Aizenman, A. Elgart, S. Naboko, J. H. Schenker, and G. Stolz, Moment analysis for localization in random Schrödinger operators, Invent. Math. 163 (2006), no. 2, 343-413.

[3] M. Aizenman and G. M. Graf, Localization bounds for an electron gas, Journal of Physics A: Mathematical and General 31 (1998), no. 32, 6783 .

[4] M. Aizenman, J. H. Schenker, R. M. Friedrich, and D. Hundertmark, Finite-volume fractional-moment criteria for Anderson localization, Comm. Math. Phys. 224 (2001), no. 1, 219-253. Dedicated to Joel L. Lebowitz.

[5] A. Avila, J. You, and Q. Zhou, Sharp phase transitions for the almost Mathieu operator, Duke Math. J. 166 (2017), no. 14, 2697-2718.

[6] J. Avron and B. Simon, Singular continuous spectrum for a class of almost periodic Jacobi matrices, Bull. Amer. Math. Soc. (N.S.) 6 (1982), no. $1,81-85$.

[7] J. Bourgain and S. Jitomirskaya, Anderson localization for the band model, in: Geometric Aspects of Functional Analysis, Vol. 1745 of Lecture Notes in Math., 67-79, Springer, Berlin (2000).

[8] J. Bourgain and S. Jitomirskaya, Continuity of the Lyapunov exponent for quasiperiodic operators with analytic potential, J. Statist. Phys. 108 (2002), no. 5-6, 1203-1218. Dedicated to David Ruelle and Yasha Sinai on the occasion of their 65 th birthdays.

[9] R. Del Rio, S. Jitomirskaya, Y. Last, and B. Simon, What is localization?, Physical Review Letters 75 (1995), no. 1, 117.

[10] F. Delyon, H. Kunz, and B. a. Souillard, One-dimensional wave equations in disordered media, J. Phys. A 16 (1983), no. 1, 25-42. 
[11] L. Ge and X. Zhao, Exponential dynamical localization in expectation for the one dimensional Anderson model, J. Spectr. Theory to appear.

[12] F. Germinet and S. Jitomirskaya, Strong dynamical localization for the almost Mathieu model, Rev. Math. Phys. 13 (2001), no. 6, 755-765.

[13] A. Y. Gordon, S. Jitomirskaya, Y. Last, and B. Simon, Duality and singular continuous spectrum in the almost Mathieu equation, Acta Math. 178 (1997), no. 2, 169-183.

[14] S. Jitomirskaya and H. Krüger, Exponential dynamical localization for the almost Mathieu operator, Comm. Math. Phys. 322 (2013), no. 3, $877-882$.

[15] S. Jitomirskaya and W. Liu, Universal hierarchical structure of quasiperiodic eigenfunctions, Ann. of Math. (2) 187 (2018), no. 3, 721776 .

[16] S. Jitomirskaya and W. Liu, Universal reflective-hierarchical structure of quasiperiodic eigenfunctions and sharp spectral transition in phase, preprint (2018), arXiv:1802.00781.

[17] S. Jitomirskaya, W. Liu and L. Mi, Palindromic argument for dynamical delocalization of almost periodic operators, preprint (2020).

[18] S. Jitomirskaya and B. Simon, Operators with singular continuous spectrum. III. Almost periodic Schrödinger operators, Comm. Math. Phys. 165 (1994), no. 1, 201-205.

[19] S. Jitomirskaya and X. Zhu, Large deviations of the Lyapunov exponent and localization for the $1 D$ Anderson model, Comm. Math. Phys. 370 (2019), no. 1, 311-324.

[20] S. Y. Jitomirskaya, Metal-insulator transition for the almost Mathieu operator, Ann. of Math. (2) 150 (1999), no. 3, 1159-1175.

[21] S. Y. Jitomirskaya and Y. Last, Anderson localization for the almost Mathieu equation. III. Semi-uniform localization, continuity of gaps, and measure of the spectrum, Comm. Math. Phys. 195 (1998), no. 1, $1-14$.

[22] H. Kunz and B. Souillard, Sur le spectre des opérateurs aux différences finies aléatoires, Comm. Math. Phys. 78 (1980/81), no. 2, 201-246. 
Dept. of Mathematics, University of California, Irvine IRvine, CA 92697-3875, USA

E-mail address: szhitomi@math.uci.edu

Mathematics 253-37, Caltech

Pasadena, CA 91125, USA

E-mail address: helge@caltech.edu

Dept. of Mathematics, University of California, Irvine IRvine, CA 92697-3875, USA

Current address:

Dept. of Mathematics, Texas A\&M University

College Station, TX 77843-3368, USA

E-mail address: liuwencai1226@gmail.com

Received December 6, 2018

Accepted June 8, 2019 